Interfaces of Propylene Carbonate

\title{
Interfaces of Propylene Carbonate
}

\author{
Xinli You, 1, a) Mangesh I. Chaudhari, 1, b) Lawrence R. Pratt, 1, (c) Noshir Pesika, 1, d) Kalika M. Aritakula, 2, () and \\ Steven W. Rick ${ }^{2, f)}$ \\ 1) Department of Chemical and Biomolecular Engineering, Tulane University, New Orleans, \\ LA 70118 \\ ${ }^{2)}$ Department of Chemistry, University of New Orleans, New Orleans, LA 70148
}

Propylene carbonate (PC) wets graphite with a contact angle of $31^{\circ}$ at ambient conditions. Molecular dynamics simulations agree with this contact angle after $40 \%$ reduction of the strength of graphite-C atom Lennard-Jones interactions with the solvent, relative to the models used initially. A simulated nano-scale PC droplet on graphite displays a pronounced layering tendency and an Aztex pyramid structure for the droplet. Extrapolation of the computed tensions of PC liquid-vapor interface estimates the critical temperature of PC accurately to about 3\%. PC molecules lie flat on the PC liquid-vapor surface, and tend to project the propyl carbon toward the vapor phase. For close PC neighbors in liquid PC, an important packing motif stacks carbonate planes with the outer oxygen of one molecule snuggled into the positively charged propyl end of another molecule so that neighboring molecule dipole moments are approximately antiparallel. The calculated thermal expansion coefficient and the dielectric constants for liquid PC agree well with experiment. The distribution of PC molecule binding energies is closely Gaussian. Evaluation of the density of the coexisting vapor then permits estimation of the packing contribution to the $\mathrm{PC}$ chemical potential, and that contribution is about $2 / 3 \mathrm{rds}$ of the magnitude of the contributions due to attractive interactions, with opposite sign.

\section{INTRODUCTION}

Unique properties of nanomaterials, and specifically of electrochemical double-layer capacitors (EDLC) based on carbon nanotube (CNT) forests,,$\frac{1}{,}$ arise from their large surface areas. Accurate descriptions of interfaces is an important challenge for modeling. Here we considered propylene carbonate (PC: FIG.11) as a solvent for electrochemical double-layer capacitors, reporting experimental and molecular simulation results on PC interfaces. This validation is preparatory to direct simulation of dynamical filling and performance of CNT-based EDLCs.

Differential filling of a charged nanotube forest from a bulk electrolyte solution is expected to be sensitive to the balance of attractive intermolecular interactions. A possible concern for the first simulations of CNTbased EDLCs was that models of the PC solvent had not been parameterized to describe contact with carbon electrodes. ${ }^{2,3}$ An initial molecular dynamics calculation showed complete spreading of the modelled liquid $\mathrm{PC}$ on graphite, $\stackrel{4}{-}$ in disagreement with experimental observation of a PC droplet on graphite reported below. That experience ${ }^{\underline{5}}$ suggested the advantage of studying PC interfaces in validation of simulation models for electrochemical applications. The present work adjusts the Lennard-Jones interactions with the graphitic carbon to agree with the observed contact angle.

Propylene carbonate is a non-reactive, low-toxicity, aprotic, highly polar dielectric solvent widely used

\footnotetext{
a) Electronic mail: xyou@tulane.edu

b) Electronic mail: chaudhari.84@gmail.com

c) Electronic mail: lpratt@tulane.edu

d)Electronic mail: npesika@tulane.edu

e)Electronic mail: karitaku@uno.edu

f)Electronic mail: srick@uno.edu
}

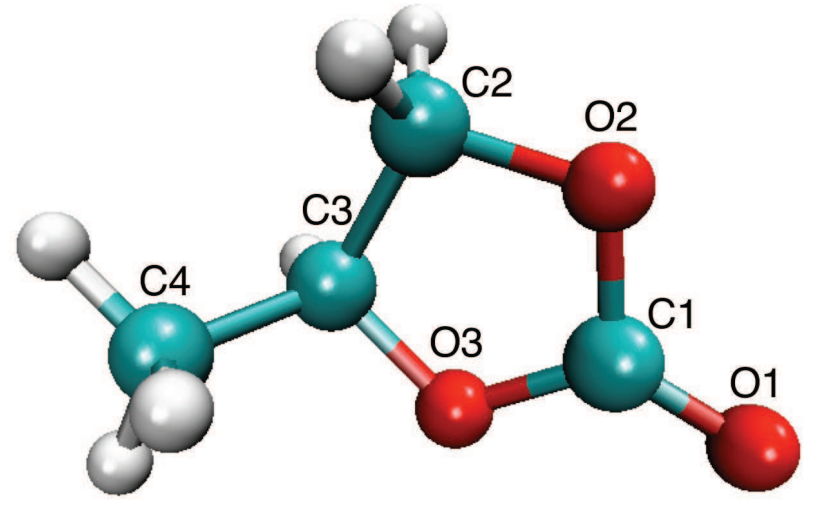

FIG. 1. Propylene carbonate (PC) enantiomer with atom labelling used in this paper.

in nonaqueous electrochemical systems. Simulation of interfaces of non-aqueous solvents for electrolytes, acetonitrile $e^{6.7}$ and propionitrile,,$\frac{8}{,}$ have been previously carried-out. Helpful molecular simulations $3,9,10$ of the uniform liquid propylene carbonate solvent are also available. A variety of molecular dynamics results are discussed below, and technical details of the methods are collected in the Appendix (Sec IV).

\section{RESULTS}

\section{A. Droplet-on-graphite contact angle}

The observed contact angle (FIG. 2) is acute. This indicates good wetting and favorable PC:graphite interactions, though not the complete spreading that was obtained ${ }^{4}$ from simulation with initial models $\stackrel{2,3}{*}$ Trial- 


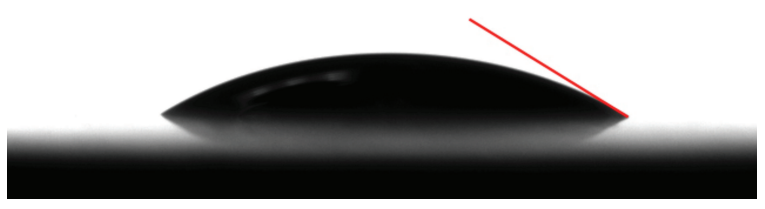

FIG. 2. PC droplet on graphite. Contact angle measurements of $5 \mu \mathrm{l}$ propylene carbonate drops on a pyrolytic graphite disc (Grade: PG-SN, Graphitestore.com) were performed under ambient conditions using a Rame-Hart contact angle goniometer. The graphite disc was sheared against a paper wipe (Kimwipe) to reveal a fresh surface. The surface was then rinsed with water and allowed to air dry. A static contact angle of $31.4 \pm 1.6^{\circ}$ was obtained from 3 trials.

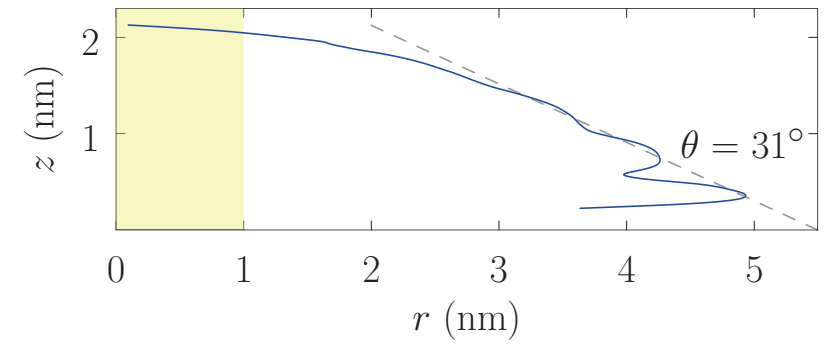

FIG. 3. Droplet silhouette, suggesting an Aztex pyramid, 12,13 The indicated contact angle is found by fitting a straight line to the region $3.0 \mathrm{~nm}<r<4.2 \mathrm{~nm}$. The shaded region identifies the cylinder used to investigate the layering of the mean density in FIG. 4 .

and-error (Sec. IV A) found a simulation contact angle of about $31^{\circ}$ with a $40 \%$ lowering of the strength of Lennard-Jones interactions associated with the $\mathrm{C}$ atoms of the graphite substrate (FIG. 33).

Simulations of water on graphite surfaces show that changing the number of water molecules from 1000 to over 17,000 has only a small effect on the contact angle.11 A droplet size dependences of such results are often ascribed to a line-tension effect. The fine structure observed here in the three-phase contact region here would make definition and further investigation of a triple line complicated. Together with the distinctly non-spherical molecular shape and packing pattern (studied below), interesting structural transitions of PC molecules near charged electrodes are expected; those issues should be the subject of future studies.

The millimeter length scales of the experimental observation differ by $10^{6}$ from nanometer length scales treated by the calculation. Pore radii for ECDL capacitors are in the nanometer range. Therefore, the nano-scale fine-structure of the molecular dynamics result (FIG. 4) should be relevant to the anticipated studies of EDCLs.

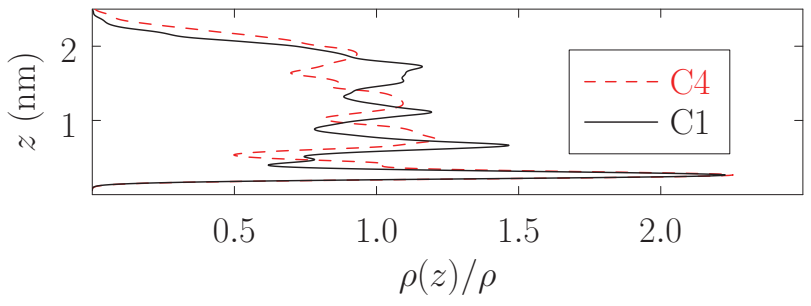

FIG. 4. The density, relative to the coexisting liquid density, in the central plug of material indicated by the shaded region of FIG. 3. Atom layers nearest to the graphite are concordant. The density oscillations of $\mathrm{C} 1$ and $\mathrm{C} 4$ atoms conflict at larger distances from the graphite, and $\mathrm{C} 4$ prevails furthest from the graphite.

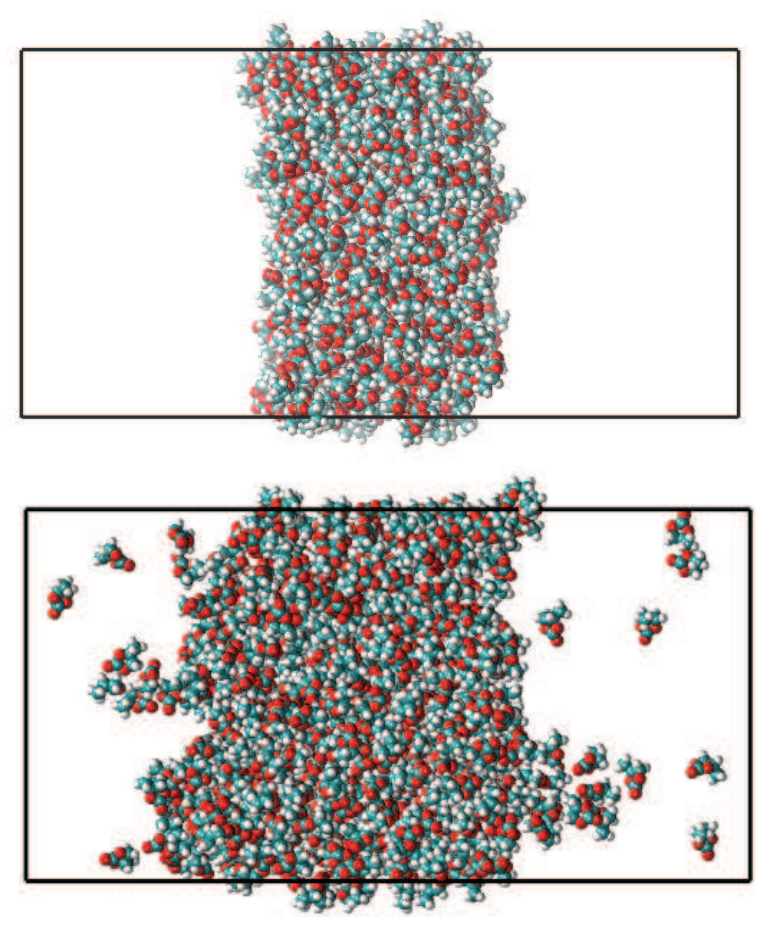

FIG. 5. Slab geometry, upper configuration from $T=300 \mathrm{~K}$ simulation, lower configuration from $T=600 \mathrm{~K}$ simulation.

\section{B. Liquid-vapor interfacial tensions}

Surface tensions were evaluated from simulations in slab geometry (FIG. 5] at several temperatures. The PC liquid-vapor interfacial tensions (FIG. 6) agree accurately with experimental values (TABLE I) at moderate temperatures. The simple formula $\gamma(T) \sim \gamma_{0}\left(\frac{T-T_{c}}{T_{c}}\right)^{5 / 4}$, utilizing an approximate estimate of the critical exponent, extrapolates to $\gamma \rightarrow 0$ at $T_{c} \approx 740 \mathrm{~K}$ (FIG. 6). 
TABLE I. Experimental surface tension at $20 \mathrm{C}$ is 41.1 dyne $/ \mathrm{cm} \cdot{ }^{14}$ Vapor densities $\rho_{\text {vap }}$ were obtained from FIG. 13 utilizing WHAM calculations at the lowest temperature.

\begin{tabular}{|c|c|c|c|c|c|c|}
\hline$T(\mathrm{~K})$ & $\rho_{\text {liq }}\left(\mathrm{nm}^{-3}\right)$ & $\rho_{\text {vap }}\left(\mathrm{nm}^{-3}\right)$ & $-k T \ln \left(\frac{\rho_{\text {liq }}}{\rho_{\text {vap }}}\right)(\mathrm{kcal} / \mathrm{mol})$ & $\rho_{\text {vap }} k T($ bar $)$ & $p(\text { bar })^{15}$ & $\gamma($ dyne $/ \mathrm{cm})$ \\
\hline 300 & 7.11 & $1.6 \mathrm{e}-6$ & -9.2 & $6.2 \mathrm{e}-5$ & $7.6 \mathrm{e}-5$ & 40.8 \\
\hline 400 & 6.46 & $4.8 \mathrm{e}-4$ & -7.6 & $2.6 \mathrm{e}-2$ & $2.8 \mathrm{e}-2$ & 29.9 \\
\hline 600 & 4.93 & $7.0 \mathrm{e}-2$ & -5.1 & 5.8 & 4.9 & 9.7 \\
\hline
\end{tabular}

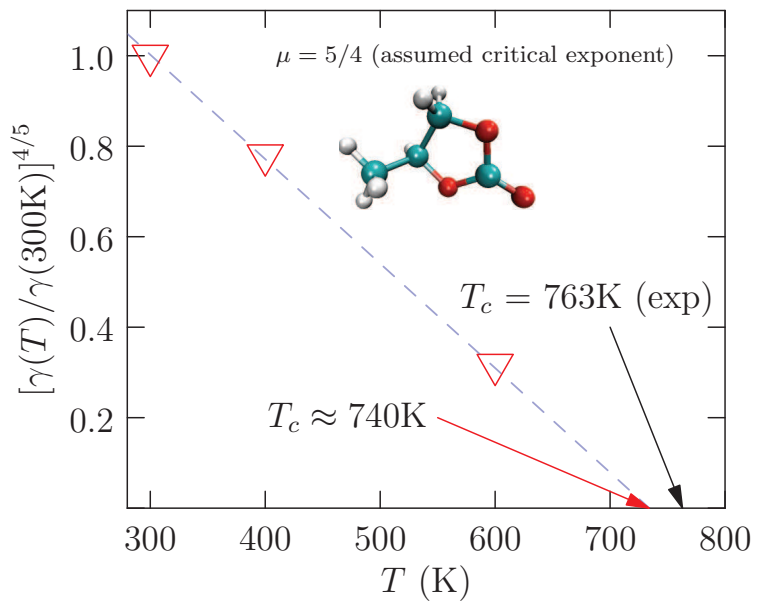

FIG. 6. $\quad$ Surface tensions $\gamma=$ $\frac{1}{2} \int\left\{p_{z z}(z)-\frac{1}{2}\left[p_{x x}(z)+p_{y y}(z)\right]\right\} \mathrm{d} z$. The experimental critical temperature ${ }^{15}$ is $T_{\mathrm{c}}=763 \mathrm{~K}$, whereas the triple temperature is about $T_{\text {triple }} \approx 220 \mathrm{~K}$.

\section{Liquid-vapor interfacial structure}

For the lowest temperatures considered here, the interfacial profiles of the atomic densities are distinctive (FIG. 7): non-monotonic except for the density of the propyl carbon (C4). The coincidence of the positive lip in atom densities other than $\mathrm{C} 4$ with the deficit in the C4 density suggests that the PC molecule lies approximately flat on this interface while projecting $\mathrm{C} 4$ further toward the vapor. Direct interrogation of the orientations of molecules with $z_{\mathrm{C} 1}>1.5 \mathrm{~nm}$ (FIG. 7) confirms this view (FIG. 8).

\section{Structure of the coexisting liquid}

The atom-atom intermolecular radial distribution functions (FIGS. 9 and 10) supplement the view that an important packing motif stacks carbonate planes of close PC neighbors with the outer (O1) oxygen of one molecule snuggled into the positively charged propyl end of another molecule so that neighboring molecule dipole moments are approximately antiparallel. Such an arrangement is similar to the known crystal structure of ethylene carbonate, $\stackrel{17}{ }$ and to the $\mathrm{PC}$ dimer structures

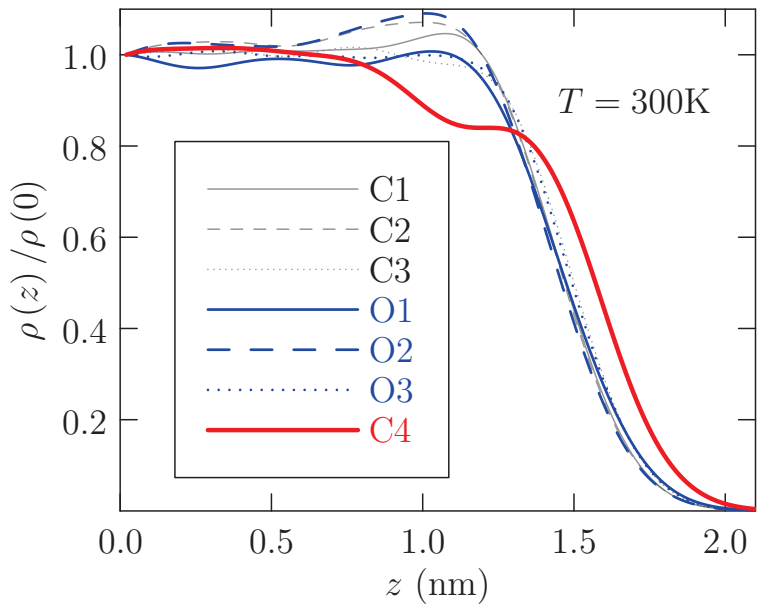

FIG. 7. The carbonate plane lies flat on the surface, projecting $\mathrm{C} 4$ from the liquid toward the vapor phase.

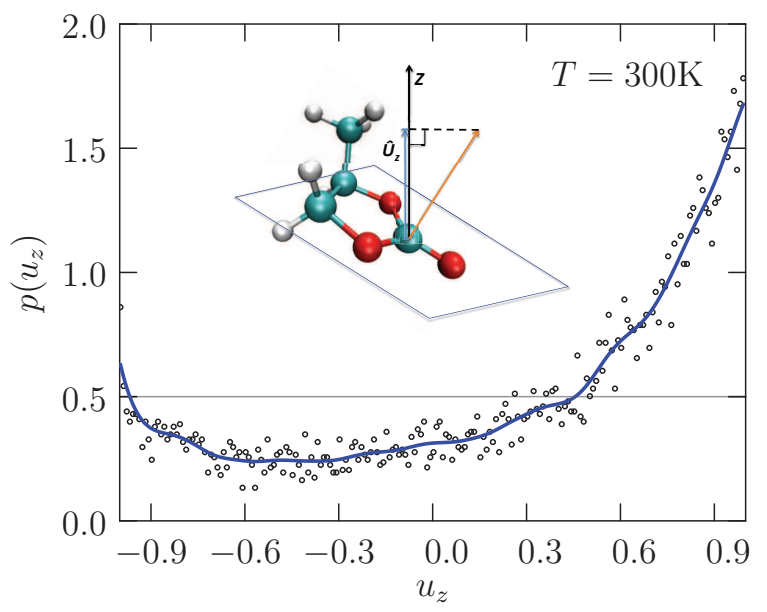

FIG. 8. For molecules with $z_{\mathrm{C} 1}>1.5 \mathrm{~nm}$ (FIG. 7), the probability density for projection of unit vector normal to the carbonate $\left(-\mathrm{CO}_{2}-\right)$ plane onto the $z$-axis, perpendicular to the interface. In this interfacial layer, the most probable orientation aligns the carbonate plane parallel with the plane of the interface, with the $\mathrm{C} 4$ methyl group extended toward the vapor phase. $u_{z}>0.5\left(\theta<60^{\circ}\right)$ for about $50 \%$ of interfacial PC molecules. 


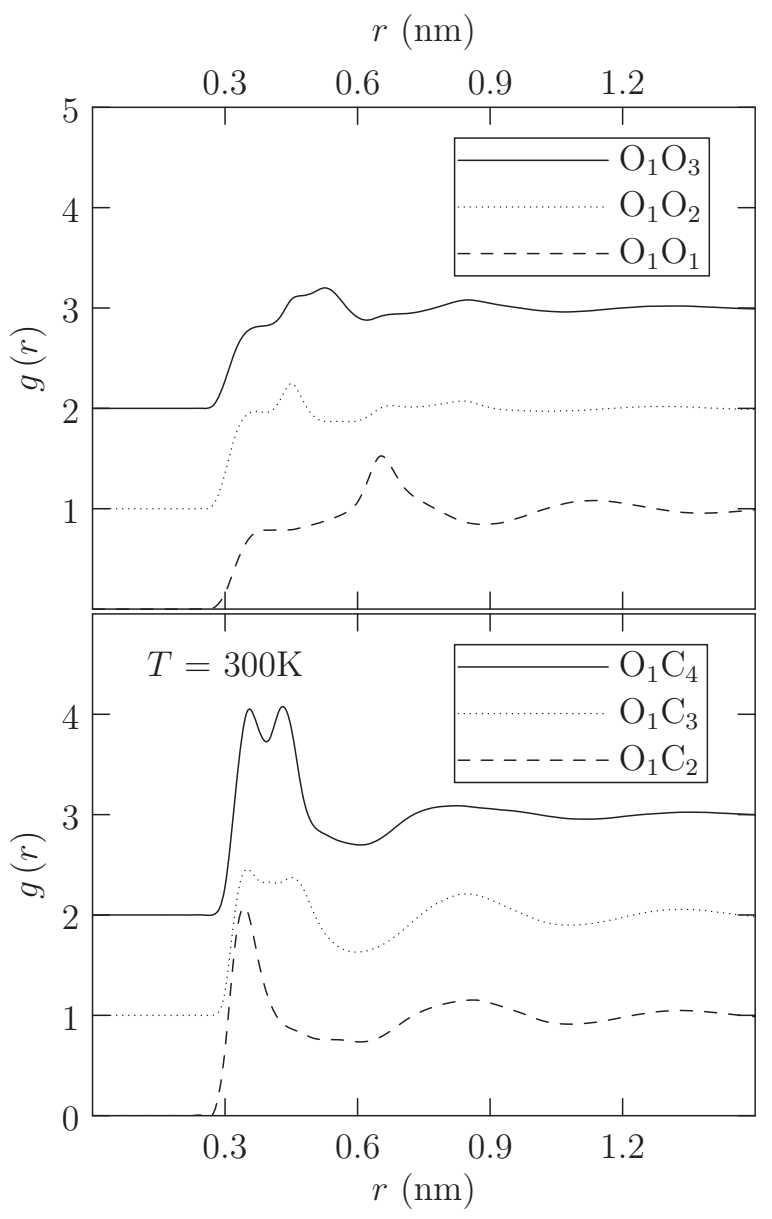

FIG. 9. Structured close-contacts involve negatively charged O1 with the opposite end of the PC molecule.

established by electronic structure calculations $\underline{10}$

On the basis of the $\mathrm{C} 1 \mathrm{C} 1$ joint distribution (FIG. 10), the nearest two neighbors of a $\mathrm{C} 1$ are physically distinct, imperfectly stacked on top and bottom of the carbonate plane. The closest $\mathrm{C} 1$ neighbor of a $\mathrm{C} 1$ atom is most probably at a radial displacement of $0.38 \mathrm{~nm}$ (FIG. 10). The observed distribution of the closest $\mathrm{C} 1$ neighbor of a $\mathrm{C} 1$ atom (FIG. 10) is more strongly peaked than the simple Poisson-based estimate 16

The dielectric constant of uniform PC liquid implied by these simulations (FIG. 11) agrees satisfactorily with experiment.

\section{E. Balance of excluded volume and attractive interactions}

Thermodynamic energies for liquid PC may be analyzed on the basis of quasi-chemical theory $\underline{20} \underline{\underline{24}}$ in which the paramount goal is molecular-scale physical clarity from thermodynamic characteristics. The quasi-chemical approach focuses on binding energies, $\varepsilon=U(N)-U(N-$ 1) $-U(1)$, of individual molecules and introduces a con-

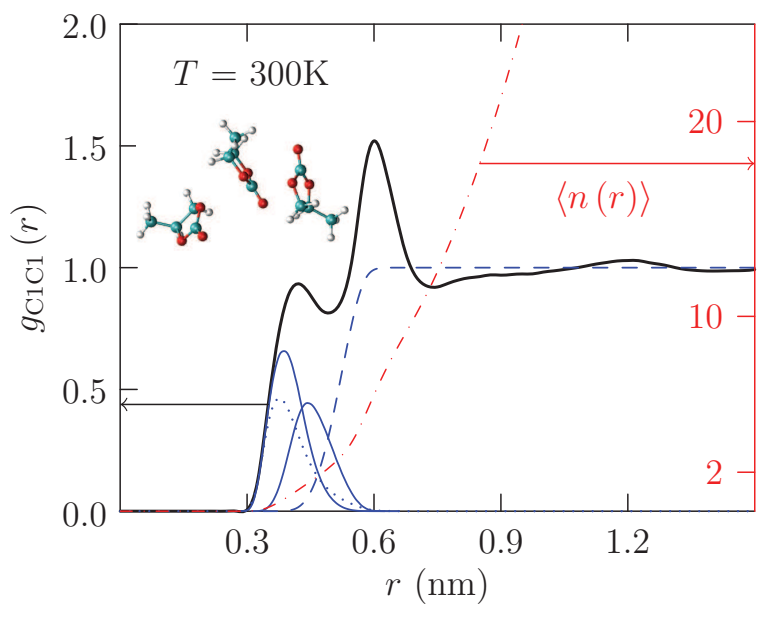

FIG. 10. Taking $\mathrm{C} 1$ as the center of the PC molecule, this characterizes the packing of centers, with molecular coordination numbers of $10-12$. The solid blue curves are the radial distribution functions for the closest, and $2 n d$-closest $\mathrm{C} 1$ neighbors of a $\mathrm{C} 1$ atom, peaked at $0.38 \mathrm{~nm}$ and $0.44 \mathrm{~nm}$, respectively. The dotted curve is the estimate of the radial distribution of the closest $\mathrm{C} 1$ neighbor of a $\mathrm{C} 1$ atom based on the Poisson approximation. ${ }^{16}$ The dashed blue curve is the probability, with median point of about $0.52 \mathrm{~nm}$, that a central $\mathrm{C} 1$ atom has more than two (2) $\mathrm{C} 1$ neighbors within that radius. The closest $\mathrm{C} 1-\mathrm{C} 1$ contacts are associated with stacking, with substantial disorder, of carbonate planes as suggested by the embedded molecular graphic.

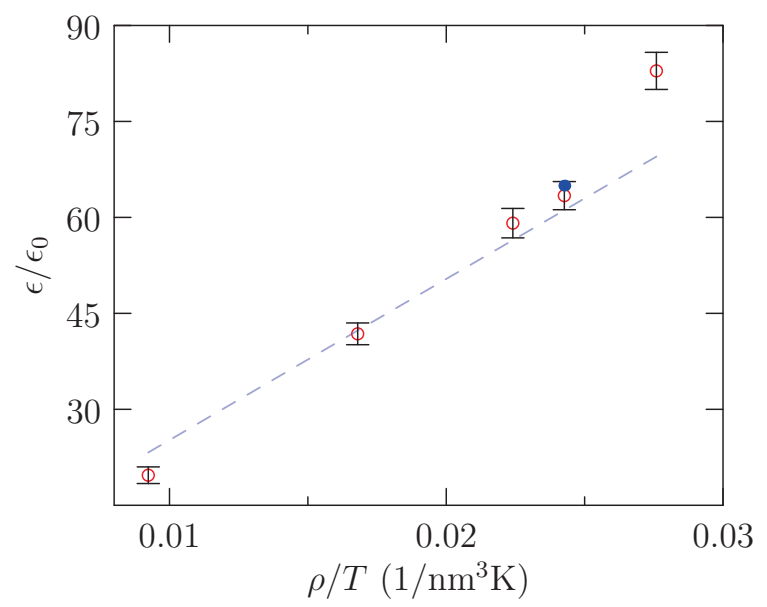

FIG. 11. Dielectric constant of model propylene carbonate, evaluated following standard simulation methods. 3,18 The calculation treated 600 PC molecules under periodic boundary conditions. Resulting values were averaged from the $40 \mathrm{~ns}$ production trajectories at constant pressure of $1 \mathrm{~atm}$. The solid dot is the measurement of Côte, et al. ${ }^{19}$ at $25 \mathrm{C}$. Numerical values are given in TABLE[I] 
ditioning based on definition of an indicator function,, 25 $\chi$, so that $\chi=1$ defines a logical condition or constraint that physically simplifies that statistical thermodynamic problem. 24

For example, if close neighbors set particularly important or complicated or chemical interactions, then the logical $\chi=1$ can indicate the absence of neighbors in a specified local region. Indeed, for numerous applications to liquid water, ${ }^{22-24,26-29} \chi=1$ indicates that there are no O-atoms of bath molecules within a specified radius from the O-atom of a distinguished water molecule. Then $\chi=0$ if any solvent molecule $i s$ present in that innershell. For the application to $\mathrm{CF}_{4}(\mathrm{aq})^{20}, \chi=0$ if any water molecule is in defined van der Waals contact with the polyatomic $\mathrm{CF}_{4}$ solute.

With such a indicator function $\chi$ specified, the partial molar Gibbs free energy in excess over the ideal contribution, $\mu^{(\mathrm{ex})}=\mu-\mu^{\text {ideal }}$, can be expressed as

$$
\begin{aligned}
\beta \mu^{(\text {ex })}=-\ln \langle\langle\chi\rangle\rangle_{0} & \\
& +\ln \int \mathrm{e}^{\beta \varepsilon} P(\varepsilon \mid \chi=1) \mathrm{d} \varepsilon \\
& +\ln \langle\chi\rangle,
\end{aligned}
$$

with $\beta^{-1}=k_{\mathrm{B}} T$. A principal virtue of this formulation ${ }^{24}$ is that is subsumes a van der Waals picture of the solvation without requiring that intermolecular interactions of different types be expressed is some specific format. We will use this formulation here to distinguish packing contributions from longer-ranged interactions that are attractive on balance. The notation $\langle\ldots\rangle$ indicates the usual average over the thermal motion of the system. The notation $\langle\langle\ldots\rangle\rangle_{0}$ indicates the average over the thermal motion of the system together with an additional molecule with no interaction between them, thus the subscript $0 .\langle\langle\chi\rangle\rangle_{0}$ is then the probability that the defined inner-shell is empty in the case that interactions between the distinguished molecule and the solution are absent. That contribution thus gauges the free energy cost of finding space for positioning the additional PC molecule in the liquid.

More broadly, these formalities follow from the identity 22,27

$$
\frac{\left\langle\mathrm{e}^{\beta \varepsilon} \chi\right\rangle}{\langle\chi\rangle}=\mathrm{e}^{\beta \mu^{(\mathrm{ex})}} \frac{\langle\langle\chi\rangle\rangle_{0}}{\langle\chi\rangle}
$$

which is a disguised expression of the rule of averages. 30,31 This broader observation Eq. (2), might permit design of weight functions $\chi$ for improved numerical performance. 22

$P(\varepsilon \mid \chi=1)$ that appears in Eq. (1) is the probability distribution of the binding energies, and is conditional on an empty inner-shell. That conditioning can make $P(\varepsilon \mid \chi=1$ ) simple enough that a Gaussian (or normal) approximation suffices $, 26,32$ The observed behavior of $P(\varepsilon)$ (FIG. 12) already suggests that possibility. Ac-

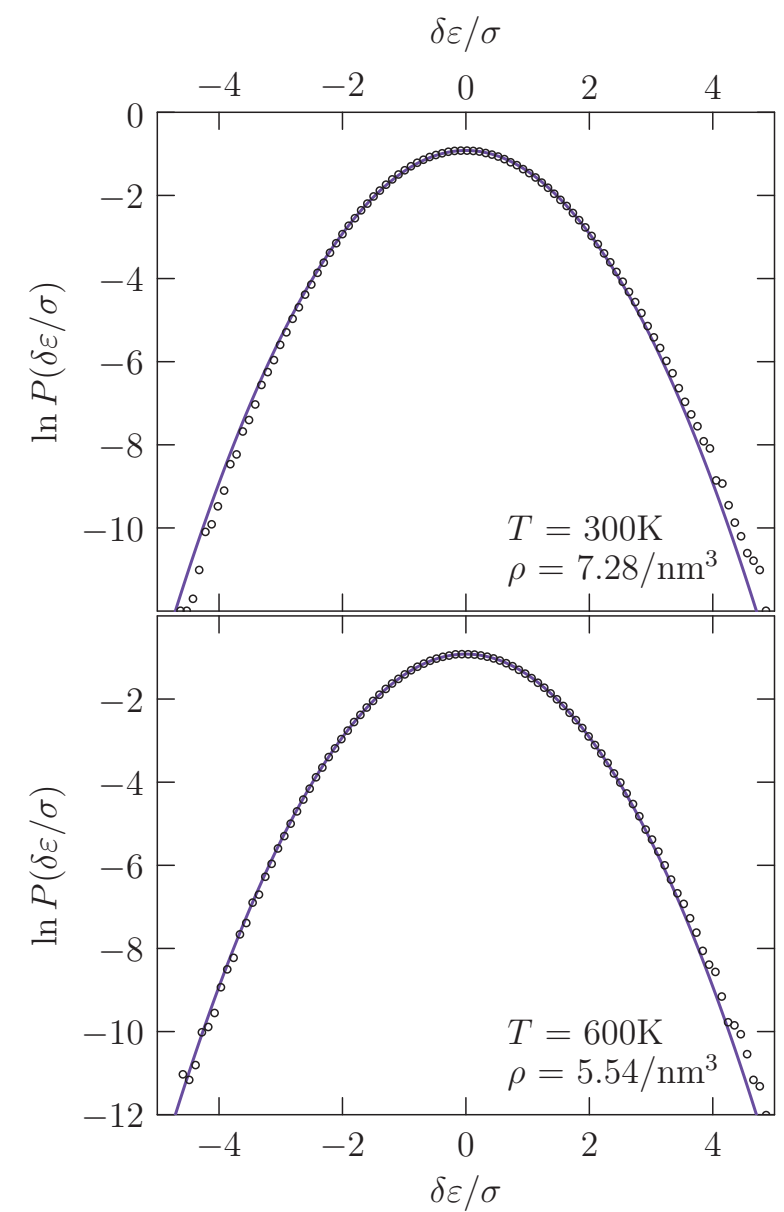

FIG. 12. Binding energies, centered $(\delta \varepsilon=\varepsilon-\langle\varepsilon\rangle)$ and scaled $\left(\sigma^{2}=\left\langle\delta \varepsilon^{2}\right\rangle\right)$, are approximately normally distributed. The slight super-gaussian behavior on the crucial right-side of these plots is the signature of repulsive intermolecular interactions.

cepting that Gaussian approximation

$$
\begin{aligned}
\beta \mu^{(\mathrm{ex})} \approx- & \ln \langle\langle\chi\rangle\rangle_{0} \\
+\beta\langle\varepsilon \mid \chi=1\rangle+\beta^{2}\left\langle\delta \varepsilon^{2} \mid \chi=1\right\rangle & / 2 \\
& +\ln \langle\chi\rangle .
\end{aligned}
$$

For conceptual clarity, let us discuss how definition of $\chi$ might be approached. $\chi=1$ corresponds to the absence of van der Waals contact of the distinguished PC molecule with the solution. We might chose to define $\chi$ by assigning van der Waals radii for all atom types. The results of FIGS. 9 and 10, characterizing close atom-atom pair distances, and particularly distance ordered contributions of FIG. 10 would be directly relevant for that. We would adjust those radii assignments to realistically large values, while targeting values of $\langle\chi\rangle$ not too different from one.

With this conceptual background, Eq. (3) becomes

$$
\beta \mu^{(\mathrm{ex})} \approx-\ln \langle\langle\chi\rangle\rangle_{0}+\beta\langle\varepsilon\rangle+\beta^{2}\left\langle\delta \varepsilon^{2}\right\rangle / 2 .
$$


This result is interesting for several reasons. Though it is suggestive of a van der Waals treatment, it is not limited to a first-order mean-field contribution, and additionally the assessment of attractive interaction is made on the basis of observations for the case that those interactions are actually operating. Nevertheless, the packing contribution $-\ln \langle\langle\chi\rangle\rangle_{0}$ is typically difficult to calculate directly.

To quantitatively characterize the effort to obtain that packing contribution, here we focus on getting an estimate on the basis of the present methods. With observation of the density of the vapor phase (FIG. 13), we can get the desired free energy on the saturation curve $\underline{\underline{31}}$

$$
\beta \mu_{\text {liq }}^{(\mathrm{ex})}=\beta \mu_{\mathrm{vap}}^{(\mathrm{ex})}-\ln \left(\frac{\rho_{\mathrm{liq}}}{\rho_{\mathrm{vap}}}\right) .
$$

Our intention (tested below) is to assume that the vapor phase is approximately ideal, $\beta \mu_{\text {vap }}^{(\mathrm{ex})} \approx 0$. The result Eq. (5) applies to the coexisting liquid. For the thermodynamic states of TABLE II at the same temperature but slightly different pressures, we apply the correction

$$
\beta \mu_{\text {liq }}^{(\text {ex })} \approx-\ln \left(\frac{\rho_{\text {liq }}}{\rho_{\text {vap }}}\right)+\beta\left(\frac{\partial \mu}{\partial p}\right)_{T} \Delta p-\ln \left(\frac{\rho}{\rho_{\text {liq }}}\right)
$$

Of course $\beta(\partial \mu / \partial p)_{T} \Delta p=\beta \Delta p / \rho_{\text {liq }}$; at $T=300 \mathrm{~K}$ (TABLE III) this term is about 0.003 and we neglect it. The rightmost term of Eq. (6) extracts the ideal contribution to the chemical potential change. Collecting all, we find that

$$
-\ln \langle\langle\chi\rangle\rangle_{0} \approx-\ln \left(\frac{\rho}{\rho_{\text {vap }}}\right)-\beta\langle\varepsilon\rangle-\beta^{2}\left\langle\delta \varepsilon^{2}\right\rangle / 2,
$$

characterizes the net effect of intermolecular excluded volumes when the vapor pressure is low.

Except for the highest temperature considered, the ideal estimate of the vapor pressure is low and close to the experimental vapor pressure (TABLE I), so the assumption of ideality of the vapor is accurate then. At $T=600 \mathrm{~K}$, the vapor pressure is substantial but the ideal estimate of the vapor pressure is still within about $20 \%$ of the experimental vapor pressure.

We therefore estimate the packing contribution $-k_{\mathrm{B}} T \ln \langle\langle\chi\rangle\rangle_{0} \approx 15 \mathrm{kcal} / \mathrm{mol}$ at $300 \mathrm{~K}$, and $14 \mathrm{kcal} / \mathrm{mol}$ at $400 \mathrm{~K}$. Thus volume exclusion effects contribute to the solvation free energies at the level of about $2 / 3 \mathrm{rds}$ of the magnitude of the attractive interactions (TABLE II), of course with opposite sign, when the vapor pressure is low. At $300 \mathrm{~K},\langle\langle\chi\rangle\rangle_{0} \approx 1 \times 10^{-11}$.

Attractive interactions stabilize the liquid of course. van der Waals attractions make a larger contribution to the mean binding energies (TABLE III) than do electrostatic interactions. Contrariwise, electrostatic contributions dominate van der Waals attractions in the variances of binding energies.

If electrostatic interactions are considered solely, then at the lowest temperature the mean and variance electrostatic contributions are roughly in the 2:1 proportion that

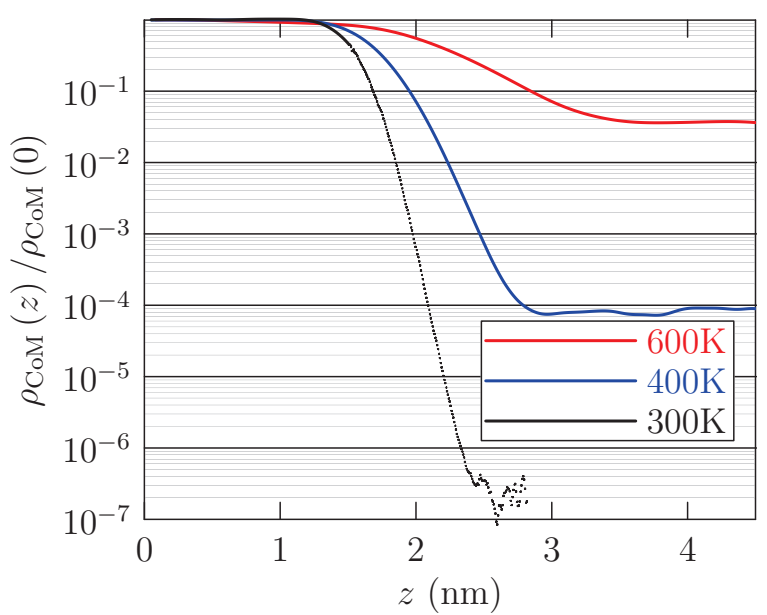

FIG. 13. The dotted results were obtained by the WHAM method in order to estimate of the vapor density for the $T=300 \mathrm{~K}$ case where that value is not satisfactorily obtained from observation of the physical simulation.

is a symptom of satisfactory Gaussian models of solvation. If the distributions of binding energies are precisely Gaussian, then

$$
\langle\langle\varepsilon\rangle\rangle_{0}=\langle\varepsilon\rangle+\beta\left\langle\delta \varepsilon^{2}\right\rangle .
$$

This follows from the general requirement that 31

$$
P(\varepsilon)=\mathrm{e}^{-\left(\varepsilon-\mu^{\mathrm{ex}}\right)} P^{(0)}(\varepsilon)
$$

where $P^{(0)}(\varepsilon)$ is the distribution of binding energies for the uncoupled case associated with the $\langle\langle\ldots\rangle\rangle_{0}$ averaging. Setting $\langle\langle\varepsilon\rangle\rangle_{0}=0$ for electrostatic interactions, then the 2:1 proportion of mean and variance contributions is clear in view of the $1 / 2$ in Eq. (4). At the highest temperature considered, the mean and variance contributions are roughly equal, so Gaussian models of solvation are not supported then. The accuracy of the inference of the packing contribution at $T=600 \mathrm{~K}$ is therefore less convincing also.

\section{CONCLUSIONS}

Propylene carbonate (PC) does not spread completely on graphite, but at ambient conditions it wets with a contact angle of $31^{\circ}$. Molecular dynamics simulations agree with this contact angle after $40 \%$ reduction of the strength of graphite-C atom Lennard-Jones interactions with the solvent, relative to the models used initially $, 2,3$ The simulation of a nano-scale PC droplet on graphite displays a pronounced layering tendency and an Aztex pyramid structure for the droplet. Extrapolation of the computed tensions of PC liquid-vapor interface estimates the critical temperature of $\mathrm{PC}$ accurately to about $3 \%$. PC molecules lie flat on the PC liquid-vapor surface, 
TABLE II. Bulk calculations at $p=1 \mathrm{~atm}, N=600$. Energies are in $\mathrm{kcal} / \mathrm{mol} . \kappa_{T}=\beta\left\langle\delta V^{2}\right\rangle /\langle V\rangle$. Experimental values ${ }^{33,34}$ for $\kappa_{T}$ at $T=25 \mathrm{C}$ are in the range $0.5-0.6 \mathrm{GPa}^{-1}$. Where not indicated explicitly, estimated statistical uncertainties are less than one (1) in the least significant digit given. The thermal expansion coefficient implied by these results is $0.94 \times 10^{-3} / \mathrm{K}$ (experimental value: $\left.:^{35} 0.845 \times 10^{-3} / \mathrm{K}\right)$.

\begin{tabular}{|c|c|c|c|c|c|}
\hline$T(\mathrm{~K})$ & $\rho\left(\mathrm{nm}^{-3}\right)$ & $\langle\varepsilon\rangle$ & $\langle\varepsilon\rangle+\frac{\beta}{2}\left\langle\delta \varepsilon^{2}\right\rangle$ & $\kappa_{T}\left(\mathrm{GPa}^{-1}\right)$ & $\epsilon / \epsilon_{0}$ \\
\hline 270 & 7.5 & -33.2 & -24.4 & 0.3 & $82.9 \pm 2.9$ \\
\hline 300 & 7.3 & -32.0 & -24.3 & 0.3 & $63.4 \pm 2.2$ \\
\hline 320 & 7.2 & -31.2 & -23.6 & 0.4 & $59.1 \pm 2.3$ \\
\hline 400 & 6.7 & -28.4 & -21.1 & 0.5 & $41.8 \pm 1.7$ \\
\hline 600 & 5.5 & -22.0 & -15.3 & 1.9 & $19.7 \pm 1.3$ \\
\hline
\end{tabular}

and tend to project the propyl carbon toward the vapor phase. Close PC neighbors stack carbonate planes with the outer (O1) oxygen of one molecule snuggled into the positively charged propyl end of another molecule so that neighboring molecule dipole moments are approximately antiparallel. The calculated thermal expansion coefficient and the dielectric constants for liquid $\mathrm{PC}$ agree well with experiment. The distribution of PC molecule binding energies is closely Gaussian. Evaluation of the density of the coexisting vapor then permits estimation of the excluded volume contribution to the PC chemical potential, and that contribution is about $2 / 3 \mathrm{rds}$ of the magnitude of the contributions due to attractive interactions, with opposite sign.

\section{ACKNOWLEDGEMENTS}

This work was supported by the National Science Foundation under the NSF EPSCoR Cooperative Agreement No. EPS-1003897, with additional support from the Louisiana Board of Regents.

\section{APPENDIX: METHODS}

The GROMACS package ${ }^{36}$ was used in all simulations. All the simulations were performed under periodic boundary conditions, electrostatic interactions calculated by the particle mesh Ewald method with a grid spacing of $0.1 \mathrm{~nm}$. Partial charges of PC are those of Ref. 3. cutoff at $0.9 \mathrm{~nm}$, and the temperature was maintained by the Nose-Hoover thermostat.

For bulk PC systems, constant NPT conditions were adopted, treating $600 \mathrm{PC}$ molecules initially positioned uniformly in a $(4.4 \mathrm{~nm})^{3}$ cubic cell utilizing Packmol. $\frac{37}{}$ The initial configuration was energy-minimized, then simulations were carried out at $T=270 \mathrm{~K}, \ldots 600 \mathrm{~K}$ with $p=1$ atm, with 1 fs integration time step, and were extended to $50 \mathrm{~ns}$ with first $10 \mathrm{~ns}$ discarded as aging.

Interfacial characteristics of liquid PC were investigated by molecular dynamics of two-phase (liquid-vapor) systems in slab geometry (FIG. 51). 600 PC molecules were positioned in a $5.3 \times 5.3 \times 10.3 \mathrm{~nm}^{3}$ cell, $T=300$, 400 , and $600 \mathrm{~K}$. After minimization, these systems were aged for 10 ns before a 10 ns production equilibrium trajectory at $300 \mathrm{~K}$. At $400 \mathrm{~K}$ and $600 \mathrm{~K}, 40 \mathrm{~ns}$ equilibrium trajectories were obtained. The interfacial tensions were assessed (FIG. 6) by differencing interfacial stresses in the standard way, averaging through the production trajectories.

Configurations were sampled from each trajectory at every $0.005 \mathrm{~ns}$ for further analysis. The electrostatic contributions to the binding energies (TABLE【) were evaluated with the GROMACS reaction field method. Those electrostatic contributions were checked against standard Ewald evaluations of electrostatic energies, and the differences were typically about $0.1 \mathrm{kcal} / \mathrm{mol}$. The differences of generalized reaction field alternatives from the other methods were substantially larger, i.e. $1-2 \mathrm{kcal} / \mathrm{mol}$.

Experimental applications almost always involve a racemic mixture of $\mathrm{PC}$. The calculations here were for the pure liquid of the enantiomer FIG. 11. This was because extensive initial calculations made that choice, $, 2,3$ and no results here are expected to be sensitive to that distinction. Explicit checking of a few cases in TABLE 1 and TABLE II confirm that no results are changed significantly for the racemic mixture. Nevertheless, we expect subsequent results to treat the racemic case.

\section{A. PC/graphite simulations}

The PC/graphite simulations used 600 PC molecules and three layers of graphite. Each layer has 9122 atoms, including 266 capping hydrogen atoms at the edges, to make a square surface of about $15.5 \mathrm{~nm}$ by $15.5 \mathrm{~nm}$. A liquid phase configuration of the $\mathrm{PC}$ molecules was placed near the graphite surface and equilibrated for 5 ns. All simulations used constant $T V N$ conditions, at $T=300 \mathrm{~K}$ and a cubic $(15.5 \mathrm{~nm})^{3}$ volume. To test how the $\mathrm{PC} /$ graphite interactions influence interfacial properties, a series of simulations with different Lennard-Jones $\varepsilon_{\mathrm{LJ}}$ values for the PC-graphite interactions were obtained, by scaling $\varepsilon_{\mathrm{LJ}}$ by factors $(0.25,0.4,0.5,0.55,0.6,0.75$ and 1). Interactions between all PC atoms and the graphite carbon atoms were all scaled. Each system was simulated for $5 \mathrm{~ns}$. For the optimal $\varepsilon_{\mathrm{LJ}}$ scaling, simulations were carried out for $10 \mathrm{~ns}$. Simulations were carried out with and without constraints on the graphite surface uti- 
lizing a harmonic restraint with force constant of 1000 $\mathrm{kJ} /\left(\mathrm{mol} \mathrm{nm} \mathrm{nm}^{2}\right)$. The simulations uses a cut-off value for equal to $0.9 \mathrm{~nm}$. Additional simulations were carried-out with a cut-off value of $1.2 \mathrm{~nm}$. The $\mathrm{PC} /$ graphite contact angle was found to insensitive to the cut-off value or the use of restraints on the surface. The indicated $40 \%$ reduction in the strength of graphite-C Lennard-Jones interactions may be the simplest adjustment that brings the simulation results into consistency with the contact angle observation.

\section{B. Droplet silhouette}

The droplet silhouette (FIG. 3) was obtained in the following way: ${ }^{11}$ On the basis of the simulation data, the mass density was binned. A two dimensional grid was used in cylindrical coordinates with $z$ perpendicular to the graphite plane and $r$ the radial coordinate. The $z$ axis contained the centroid of the droplet. The function

$$
\rho(r, z)=\frac{\rho_{\text {liq }}}{2}\left\{1-\tanh \left[\frac{r-r_{0}(z)}{d(z)}\right]\right\},
$$

which acknowledges that the $\rho_{\text {vap }} \approx 0$, was fit to the binned mass density. Thus the width of the interfacial profile, $d(z)$, and the position of the interface, $r_{0}(z)$ were obtained for each $z$ layer. Since the density exhibits distinct layering parallel to $z$ (FIG. 4), this procedure is particularly effective where analysis of the density in constant- $z$ slices is natural, for $0<z<1.9 \mathrm{~nm}$.

For $z>1.9 \mathrm{~nm}$, where the $0<r<2 \mathrm{~nm}$ portion of the fluid interface is roughly parallel to the $z=$ constant (graphite) surface, constant- $r$ slices of the density were similarly fit to the function

$$
\rho(r, z)=\frac{\rho_{\mathrm{liq}}}{2}\left\{1-\tanh \left[\frac{z-z_{0}(r)}{d(r)}\right]\right\} .
$$

In view of FIG. 4, this describes the outer (largest $r$ ) behavior, where the density is decreasing through $\rho_{\text {liq }} / 2$. The two approaches give the same values near a crossing point $r \approx 2 \mathrm{~nm}$. Combing these two fits gives FIG. 3 .

\section{Windowed sampling for calculations of coexisting vapor densities}

The coexisting vapor densities were evaluated by stratification on the basis of the Weighted Histogram Analy-

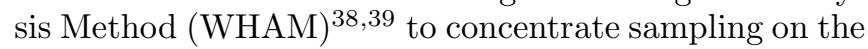
low densities of the vapor phase. Windowed calculations were performed with $600 \mathrm{PC}$ molecules at temperatures $300 \mathrm{~K}$ and $400 \mathrm{~K}$ adopting the methods described above. Initial configuration for each window was obtained by pulling a PC molecule from the center of mass of the slab to deep into vapor phase at constant rate of $0.01 \mathrm{~nm} / \mathrm{ps}$. In the $300 \mathrm{~K}$ case, 15 windows, spaced by $0.1 \mathrm{~nm}$ in bulk liquid phase and $0.05 \mathrm{~nm}$ near interface and vapor phase, were treated. Trajectories of $5 \mathrm{~ns} /$ window (after $1 \mathrm{~ns}$ of minimization and 5 ns of aging) were used to reconstruct density profiles. For the $400 \mathrm{~K}$ calculation, 41 windows of $0.1 \mathrm{~nm}$ uniform spacing were used.

The harmonic potential energy $U=k\left(z-z_{0}\right)^{2}$ windowing function was employed, with $z$ is the instantaneous distance of the center of mass of the pulled PC molecule from center of mass of the slab and $z_{0}$, the designated minimum of $U$, identifying the window position. $k=4000 \mathrm{~kJ} / \mathrm{mol} / \mathrm{nm}^{2}$ (for $300 \mathrm{~K}$ ) and $k=6000$ $\mathrm{kJ} / \mathrm{mol} / \mathrm{nm}^{2}$ (for $400 \mathrm{~K}$ ).

\section{Liquid PC dielectric constant}

The dielectric constant of uniform PC liquid is evaluated following standard simulation methods. 3,18 The calculation treated 600 PC molecules under periodic boundary conditions. Resulting values were averaged from the $40 \mathrm{~ns}$ production trajectories at constant pressure of 1 atm. 
${ }^{1}$ A. C. Dillon, Chem. Rev. 110, 6856 (2010).

${ }^{2}$ L. Yang, B. H. Fishbine, A. Migliori, and L. R. Pratt, J. Am. Chem. Soc 131, 12373 (2009).

${ }^{3}$ L. Yang, B. H. Fishbine, A. Migliori, and L. R. Pratt, J. Chem. Phys. 132, 044701 (2010).

${ }^{4}$ G. G. Hoffman, R. Mariano, N. Pesika, L. R. Pratt, S. W. Rick, and X. You, "Simulation of Propylene Carbonate as a Solvent for Electrochemical Double-layer Capacitors," Tech. Rep. (Proceedings of Louisiana EPSCoR RII LA-SiGMA 2011 Symposium, 2011) proceedings of Louisiana EPSCoR RII LA-SiGMA 2011 Symposium.

${ }^{5}$ X. You, K. M. Aritakula, N. Pesika, L. R. Pratt, and S. W. Rick, "Simulation of Propylene Carbonate Solutions for Electrochemical Double-layer Capacitors," Tech. Rep. (Proceedings of Louisiana EPSCoR RII LA-SiGMA 2012 Symposium, 2012) proceedings of Louisiana EPSCoR RII LA-SiGMA 2012 Symposium. ${ }^{6}$ S. Paul and A. Chandra, J. Phys. Chem. B 109, 20558 (2005).

${ }^{7}$ Z. Hu and J. D. Weeks, J. Phys. Chem. C 114, 10202 (2010).

${ }^{8}$ S. Liu, Z. Hu, J. D. Weeks, and J. T. Fourkas, J. Phys. Chem. C 116, 4012 (2012).

${ }^{9}$ J. C. Soetens, C. Millot, B. Maigret, and I. Bako, J. Mol. Liq. 92, 201 (2001).

${ }^{10}$ L. B. Silva and L. C. G. Freitas, J. Mol. Struct.-Theochem 806 (2007).

${ }^{11}$ R. Werder, J. H. Walther, R. L. Jaffe, T. Halicioglu, and P. Koumoutsakos, J. Phys. Chem. B 107, 1345 (2003).

${ }^{12}$ F. Heslot, N. Fraysee, and A. M. Cazabat, Nature 338, 640 (1989).

${ }^{13}$ P.-G. deGennes, F. Borchard-Wyart, and D. Quéré, Capillarity and Wetting Phenomena (Springer, 2004).

${ }^{14}$ A. W. Adamson and A. P. Gast, in Physical chemistry of surfaces (Wiley) 6th ed.

${ }^{15}$ G. M. Wilson, D. M. Von Niederhausern, and N. F. Giles, J. Chem. \& Eng. Data 47, 761 (2002).

${ }^{16}$ P. Zhu, Y. You, L. R. Pratt, and K. D. Papadopoulos, J. Chem. Phys. 134, 054502 (2011).

${ }^{17}$ C. J. Brown, Acta Cryst. 7, 92 (1954).

${ }^{18}$ Y. Wu, H. L. Tepper, and G. A. Voth, J. Chem. Phys. 124, 024503 (2006).

${ }^{19}$ J.-P. Côte, D. Brouillette, J. E. Desnoyers, J.-F. Rouleau, J.-M. St-Arnaud, and G. Perron, J. Soln. Chem. 25, 1163 (1996).
${ }^{20}$ D. Asthagiri, H. S. Ashbaugh, A. Piryatinski, M. E. Paulaitis, and L. R. Pratt, J. Am. Chem. Soc 129, 10133 (2007).

${ }^{21}$ D. Asthagiri, P. D. Dixit, S. Merchant, M. E. Paulaitis, L. R. Pratt, S. B. Rempe, and S. Varma, Chem. Phys. Letts. 485, 1 (2010).

${ }^{22}$ S. Chempath, L. R. Pratt, and M. E. Paulaitis, J. Chem. Phys. 130, 054113(1 (2009).

${ }^{23}$ S. Chempath and L. R. Pratt, J. Phys. Chem. B 113, 4147 (2009).

${ }^{24}$ D. M. Rogers, D. Jiao, L. R. Pratt, and S. B. Rempe, Ann. Rep. Comp. Chem. 8, 71 (2012).

${ }^{25}$ N. G. van Kampen, Stochastic Processes in Physics and Chemistry (Elsevier, 1992).

${ }^{26}$ J. Shah, D. Asthagiri, L. Pratt, and M. Paulaitis, J. Chem. Phys. 127, 144508 (1 (2007).

${ }^{27}$ V. Weber, S. Merchant, and D. Asthagiri, J. Chem. Phys. 135, 181101 (2011).

${ }^{28}$ V. Weber and D. Asthagiri, J. Chem. Phys. 133, 141101 (2010).

${ }^{29}$ V. Weber, S. Merchant, P. D. Dixit, and D. Asthagiri, J. Chem. Phys. 132, 204509 (2010).

${ }^{30}$ M. E. Paulaitis and L. R. Pratt, Adv. Prot. Chem. 62, 283 (2002).

${ }^{31}$ T. L. Beck, M. E. Paulaitis, and L. R. Pratt, The Potential Distribution Theorem and Models of Molecular Solutions (Cambridge University Press, 2006).

${ }^{32}$ S. Chempath, L. R. Pratt, and M. E. Paulaitis, Chem. Phys. Letts. 487, 24 (2010).

${ }^{33}$ G. A. Bottomley and M. T. Bremers, Aust. J. Chem. 39, 1959 (1986).

${ }^{34}$ Y. Marcus and G. T. Hefter, J. Mol. Liq. 73, 61 (1997).

${ }^{35}$ H. Piekarski, K. Kubalczyk, and M. Wasiak, J. Chem. \& Eng. Data 55, 5435 (2010).

${ }^{36}$ B. Hess, C. Kutzner, D. van der Spoel, and E. Lindahl, J. Chem. Theory Comp. (2008).

${ }^{37}$ L. Martínez, R. Andrade, E. G. Birgin, and J. M. Martínez, J. Comp. Chem. 30, 2157 (2009).

${ }^{38}$ S. Kumar, D. Bouzida, R. H. Swendsen, P. A. Kollman, and J. M. Rosenberg, J. Comp. Chem. 13, 1011 (1992).

${ }^{39}$ A. Grossfield, "WHAM," Tech. Rep. (University of Rochester Medical Center, 2010) http://membrane.urmc.rochester.edu/ Software/WHAM/WHAM.html. 